PUPT-1305

\title{
Quantum Rings and Recursion Relations in 2D Quantum Gravity
}

\author{
Shamit Kachru ${ }^{\dagger}$ \\ Joseph Henry Laboratories \\ Jadwin Hall \\ Princeton University \\ Princeton, NJ 08544 USA
}

\begin{abstract}
I study tachyon condensate perturbations to the action of the two dimensional string theory corresponding to the $\mathrm{c}=1$ matrix model. These are shown to deform the action of the ground ring on the tachyon modules, confirming a conjecture of Witten. The ground ring structure is used to derive recursion relations which relate $(\mathrm{N}+1)$ and $\mathrm{N}$ tachyon bulk scattering amplitudes. These recursion relations allow one to compute all bulk amplitudes.
\end{abstract}

January 1992

$\dagger$ Supported in part by an NSF Graduate Fellowship 


\section{Introduction}

Since the discovery of the double scaling limit of random matrix models [1][2][3], the study of $D \leq 2$ quantum gravity has advanced rapidly. The most interesting of the exactly soluble models is undoubtedly the $\mathrm{c}=1$ model. The continuum analogue of this model is a two dimensional string theory, which in addition to one field theoretic degree of freedom (the massless tachyon) contains an infinite number of discrete states [4]. These states appear only at certain quantized values of the momentum, and some of them have been interpreted as remnants of the transverse string excitations in the two dimensional theory [5]. The currents which can be formed from the discrete states have also been shown to generate a $W_{\infty}$ symmetry [6],[7].

In [6], Witten has demonstrated that the ghost number 0 , conformal dimension $(0,0)$ operators form a ring (with product given by the operator product expansion) whose properties are particularly important for understanding the model. In this paper, I will show that the ring structure allows one to find recursion relations which determine the bulk tachyon scattering amplitudes. I will restrict myself to the non-compact string theory. Similar recursion relations were found in [8] using the Ward identities of the $W_{\infty}$ symmetry.

The action of the unperturbed two dimensional string theory under study is given, in conformal gauge, by:

$$
S=\frac{1}{2 \pi} \int \sqrt{\hat{g}}\left(\hat{g}^{a b} \partial_{a} \phi \partial_{b} \phi+\hat{g}^{a b} \partial_{a} X \partial_{b} X-\frac{Q}{4} \hat{R} \phi+2 \mu e^{-\sqrt{2} \phi}\right)
$$

Here, $\mathrm{Q}=2 \sqrt{2}$ and $\mu$ is the cosmological constant, $\hat{g}$ is a fiducial metric and $\hat{R}$ is its curvature scalar, $\mathrm{X}$ is a free boson, and $\phi$ is the Liouville field.

\section{Tachyon Modules of the Ground Ring}

It was shown in [6] that in the non-compact model, the ground ring is the ring generated by $a_{+}$and $a_{-}$where

$$
\begin{aligned}
& a_{+}=\left|\left(c b+\frac{i}{\sqrt{2}}(\partial X-i \partial \phi)\right)\right|^{2} e^{\frac{1}{\sqrt{2}}(i X-\phi)} \\
& a_{-}=\left|\left(c b-\frac{i}{\sqrt{2}}(\partial X+i \partial \phi)\right)\right|^{2} e^{\frac{-1}{\sqrt{2}}(i X+\phi)}
\end{aligned}
$$

It is further argued there that one should expect the ground ring generators to satisfy a relation

$$
a_{+} a_{-}=\mu
$$


heuristically on the grounds that $a_{+}, a_{-}$are analogous to $\mathrm{p}+\mathrm{q}$, p-q in the fermion field theory avatar of the matrix model [9], where (3) becomes the equation defining the fermi surface. In addition, an ansatz is given in [6] for how the relation (3) should be deformed when the action (1) is perturbed by discrete state conformal dimension $(1,1)$ moduli.

It is shown in [10] that the tachyon states of the theory fall into modules of the ring. The tachyon states are given by:

$$
T_{q}^{ \pm}=e^{\frac{1}{\sqrt{2}}[i q X+(2 \mp q) \phi]}
$$

and for vanishing $\mu$, the regular tachyon modules (the integer q tachyons are discrete states) are specified (for + tachyons) by:

$$
\begin{gathered}
a_{+} c \bar{c} T_{q}^{+}=q^{2} c \bar{c} T_{q+1}^{+}+[Q, . .] \\
a_{-} c \bar{c} T_{q}^{+}=0+[Q, . .]
\end{gathered}
$$

where $\mathrm{Q}$ is the BRST operator. The - tachyon modules are similar, with the roles of the two ring generators being reversed.

Notice in particular that the relation (3) is satisfied for $\mu=0$ on the tachyon modules. We will see that its conjectured generalizations will also be satisfied on the regular tachyon modules (this is also discussed in [10]) and that the structure of the deformed modules when the action (1) is perturbed will allow us to derive recursion relations for the tachyon correlation functions.

\section{Perturbations of $\mathrm{S}$ and the Deformed Ground Ring}

Recall that in string theory, conformal dimension $(1,1)$ operators are moduli which can be integrated over the worldsheet, and hence may be added to the Lagrangian.

Let us begin by considering the addition to the action (1) of an arbitrary + tachyon condensate:

$$
S^{\prime}=S_{\mu=0}+t_{k} \int T_{k}^{+}
$$

How are the modules (5) deformed by this perturbation to the action?

The lowest order correction to the action of $a_{+} a_{-}$on the module containing $T_{q}^{+}$comes from the order $t_{k}$ correction to the action of $a_{-}$. Expanding the $e^{-S^{\prime}}$ in the functional integral representation for correlation functions, it is apparent that this lowest order correction is:

$$
a_{-} c \bar{c} T_{q}^{+}=-\lim _{w \rightarrow 0} c \bar{c} T_{q}^{+}(0) a_{-}(w) t_{k} \int T_{k}^{+}(z) d^{2} z
$$


Performing the contractions, we see that the leading term is:

$$
\begin{aligned}
& -t_{k} \int d^{2} z|z|^{2 q+2 k-4}|z-1|^{-2 k} c \bar{c} T_{q+k-1}^{+}(0)= \\
& -\pi t_{k} \frac{\Gamma(q+k-1) \Gamma(1-q) \Gamma(1-k)}{\Gamma(2-q-k) \Gamma(q) \Gamma(k)} c \bar{c} T_{q+k-1}^{+}(0)
\end{aligned}
$$

Defining

$$
\Delta(q)=\frac{\Gamma(q)}{\Gamma(1-q)}
$$

we find:

$$
a_{-} c \bar{c} T_{q}^{+}=-t_{k} \pi \Delta(k)^{-1} \Delta(q)^{-1} \Delta(q+k-1) T_{q+k-1}^{+}
$$

This is the desired first order correction to the $a_{-}$action on the tachyon module. However, from [11] [12] we expect the correlation functions etc. of the two dimensional string theory to look simple only in terms of the renormalized tachyon field and couplings, $\tilde{T}_{q}^{+}$and $\tilde{t}_{k}$ :

$$
\begin{gathered}
\tilde{T}_{q}^{+}=\Delta(q) T_{q}^{+} \\
\tilde{t}_{k}=\pi \Delta(k)^{-1} t_{k}
\end{gathered}
$$

Interestingly, it has been determined in [13] that it is exactly with this normalization that the tachyon transforms like a typical dimension 1 field under a Virasoro sub-algebra of the algebra of the $W_{\infty}$ charges.

In terms of these renormalized fields and couplings, the deformed action of the ground ring generators on the tachyons $T_{q}^{+}$in the theory with perturbed action (6) becomes:

$$
\begin{gathered}
a_{+} c \bar{c} \tilde{T}_{q}^{+}=-c \bar{c} \tilde{T}_{q+1}^{+}+[Q, . .] \\
a_{-} c \bar{c} \tilde{T}_{q}^{+}=-\tilde{t}_{k} c \bar{c} \tilde{T}_{q+k-1}^{+}+[Q, . .]
\end{gathered}
$$

The case of interest for the ansatz of [6] regarding deformations to the fermi surface (3) is the case when $\mathrm{k}$ is positive integral, and the deformation is by one of the discrete states. In this case, the multiplicative renormalization (10) becomes singular, but proceeding with this caveat we find from (11) that:

$$
a_{+} a_{-} c \bar{c} \tilde{T}_{q}^{+}=-a_{+} \tilde{t}_{k} c \bar{c} \tilde{T}_{q+k-1}^{+}=(-1)^{k} \tilde{t}_{k}\left(a_{+}\right)^{k} \tilde{T}_{q}^{+}
$$

which implies that acting on the tachyon states $\mathrm{c} \bar{c} \tilde{T}_{q}^{+}$the ring generators satisfy the relation:

$$
a_{+} a_{-}=(-1)^{k} \tilde{t}_{k}\left(a_{+}\right)^{k}
$$

In the special case $k=0$ and denoting $\tilde{t}_{0}$ by $\mu$ this reproduces (3), confirming this part of the analogy of $2 \mathrm{~d}$ string theory with the matrix model. Notice also that by considering perturbations by $\tilde{T}_{k}^{-}$, we would find a very similar relation with the roles of $a_{-}$and $a_{+}$ reversed. 


\section{Recursion Relations for Tachyon Correlation Functions}

Using the relations $(7),(8)$, we see that in essence $a_{-}$can be used to fuse two + tachyons into a single + tachyon, while we know that for - tachyons:

$$
a_{-} T_{p}^{-}=p^{2} T_{p-1}^{-}+[Q, . .]
$$

In addition, since $a_{-}$is a conformal dimension $(0,0)$ BRST invariant operator, correlation functions with $a_{-}$insertions should be independent of the position of the $a_{-}$insertion, up to contributions coming from contact terms at the boundaries of moduli space (similar arguments were used in evaluating correlation functions with ground ring insertions in [10],[14]). I will now use this fact to find recursion relations which allow us to compute all of the bulk tachyon correlation functions in terms of the three point function, in similar spirit to [8] where Ward identities of the $W_{\infty}$ symmetry were used to find recursion relations.

Consider the correlation function

$$
B_{n, 1}\left(q_{i}, p\right)=<a_{-}(z) c \bar{c} \tilde{T}_{q_{1}}^{+}(0) c \bar{c} T_{p}^{-}(1) c \bar{c} \tilde{T}_{q_{2}}^{+}(\infty) \prod_{i=3}^{n} \int d^{2} z_{i} \tilde{T}_{q_{i}}^{+}>
$$

with $\mathrm{n}+$ tachyons, one - tachyon and a ground ring insertion. Notice that $T_{p}^{-}$remains unrenormalized, as the conservation laws tell us that it has integral momentum and the renormalization factor (10) would be infinite.

Before proceeding, it may help to discuss briefly some geometric subtleties associated with correlation functions like $B_{n, 1}$, which contain insertions of operators at non-standard values of the ghost number. In string theory, when one considers normal correlation functions of physical states which have ghost number two, the correlation functions on a genus $g$ Riemann surface with $n$ operator insertions yield integrals of top forms on $\overline{\mathcal{M}}_{g, n}$, the Deligne-Mumford compactification of the moduli space of Riemann surfaces of genus $g$ with $n$ punctures. In the case at hand, however, we are considering correlation functions of $n$ operators where $n-1$ have standard ghost number, and one has ghost number zero. Hence, we are left with an element of $H^{6 g-6+2 n-2}\left(\overline{\mathcal{M}}_{g, n}\right)$ which must be integrated over a homology cycle of codimension two in order to yield a number. One would hope that the assertions made below can be formulated in these geometrical terms by saying that for certain natural distinct choices of this homology cycle, in the specific cases at hand, one obtains the same number. I do not understand how this works in any detail, however, and it may not be the correct interpretation of the underlying geometry. 
Now, let us study $B_{n, 1}$ in the limits as $z \rightarrow 0$ and $z \rightarrow 1$. The BRST commutators which arise in moving the ground ring insertion do not contribute to the correlation function [10], so the two limits of the correlation function must be equal. However, we cannot naively use the relations (5). As we have seen, there will be contributions from contact terms fusing two tachyons into one. These can be interpreted as contributions from the BRST commutators in (5).

Taking the limit as $z \rightarrow 1$ and using (14), we find:

$$
B_{n, 1}\left(q_{i}, p\right)=p^{2}<c \bar{c} \tilde{T}_{q_{1}}^{+}(0) c \bar{c} T_{p-1}^{-}(1) c \bar{c} \tilde{T}_{q_{2}}^{+}(\infty) \prod_{i=3}^{n} \int d^{2} z_{i} \tilde{T}_{q_{i}}^{+}>
$$

where from [10] we know that any further BRST commutators on the right hand side of (14) do not contribute. The limit as $z \rightarrow 0$ is slightly more subtle, but using (7), (8) we realize that we simply pick up (n-2) contributions where the integrated tachyons are fused with $\tilde{T}_{q_{1}}^{+}$. Hence:

$$
B_{n, 1}\left(q_{i}, p\right)=\sum_{i=3}^{n} \pi<c \bar{c} \tilde{T}_{q_{1}+q_{i}-1}^{+}(0) c \bar{c} T_{p}^{-}(1) c \bar{c} \tilde{T}_{q_{2}}^{+}(\infty) \prod_{j=3, j \neq i}^{n} \int d^{2} z_{j} \tilde{T}_{q_{j}}^{+}>
$$

Thus, combining (16), (17) we see that:

$$
\begin{gathered}
p^{2}<c \bar{c} \tilde{T}_{q_{1}}^{+}(0) c \bar{c} T_{p-1}^{-}(1) c \bar{c} \tilde{T}_{q_{2}}^{+}(\infty) \prod_{i=3}^{n} \int d^{2} z_{i} \tilde{T}_{q_{i}}^{+}> \\
=\sum_{i=3}^{n} \pi<c \bar{c} \tilde{T}_{q_{1}+q_{i}-1}^{+}(0) c \bar{c} T_{p}^{-}(1) c \bar{c} \tilde{T}_{q_{2}}^{+}(\infty) \prod_{j=3, j \neq i}^{n} \int d^{2} z_{j} \tilde{T}_{q_{j}}^{+}>
\end{gathered}
$$

Denoting tachyon correlation functions with $\mathrm{n}$ plus tachyons and 1 minus tachyon as $(\mathrm{n}, 1)$ correlation functions, we see that we have a recursion relation expressing an $(n, 1)$ correlation function as a sum of $(n-1,1)$ correlation functions. It is obvious how similar arguments can be used to obtain recursion relations for $(1, n)$ correlation functions, and it was shown in [10] that ring insertion arguments of this sort also suffice to prove that all $(\mathrm{n}, \mathrm{m})$ correlation functions with $n, m \geq 2$ vanish.

It is well known that in fact the non-vanishing bulk correlation functions are constant (they are evaluated explicitly in [12]), and if we normalize the $(2,1)$ function $A_{2,1}=1$ (henceforth $A_{n, 1}$ will be used to denote the (n,1) tachyon correlation function) we can find the rest of the non-vanishing correlation functions using (18). 
From the conditions of momentum and Liouville momentum conservation, we find that in $B_{n, 1}$ in (15)

$$
|p|=n-2
$$

Hence, using (18) to find $A_{3,1}$ we see that

$$
A_{3,1}=\pi A_{2,1}=\pi
$$

which we know to be correct. Using the fact that we know $A_{n-1,1}$ to be a constant, we can determine using (18) and (19) that:

$$
A_{n, 1}=\frac{\pi}{n-2} A_{n-1,1}
$$

and using $A_{2,1}=1$ we find

$$
A_{n, 1}=\frac{\pi^{n-2}}{(n-2) !}
$$

which reproduces the results of direct computation [11],[12].

\section{Conclusion}

We have seen in this paper that the ground ring structure suffices to determine all of the non-vanishing bulk correlation functions of the two dimensional string theory (1). It will be very interesting to see if the ring structure in other two dimensional string backgrounds (e.g., the two dimensional black hole [15]) yields similar simplifications in calculating correlation functions. It would also be gratifying to obtain a deeper understanding of the geometrical meaning of the relations derived here.

\section{Acknowledgements}

I would like to thank Jacques Distler for several enlightening discussions, Igor Klebanov for pointing out that I should look for recursion relations, and Edward Witten for suggesting the problem and for his continual guidance.

\section{REFERENCES}

1. D. Gross and A. Migdal, Phys. Rev. Lett. 64 (1990), 127.

2. M. Douglas and S. Shenker, Nucl. Phys. B335 (1990), 635. 
3. E. Brezin and V. Kazakov, Phys. Lett. B236 (1990), 144.

4. B. Lian and G. Zuckerman, Phys. Lett. B254 (1991), 417.

5. A. Polyakov, Mod. Phys. Lett. A6 (1991), 635.

6. E. Witten, IAS preprint IASSNS-HEP-91/51.

7. I. Klebanov and A. Polyakov, Princeton preprint PUPT-1281.

8. I. Klebanov, Princeton preprint PUPT-1302.

9. S. Das, A. Dhar, G. Mandal, and S. Wadia, IAS preprint IASSNS-HEP-51.

10. D. Kutasov, E. Martinec, and N. Seiberg, Rutgers preprint RU-91-49.

11. D. Gross and I. Klebanov, Nucl. Phys. B359 (1991), 3.

12. P. Di Francesco and D. Kutasov, Princeton preprint PUPT-1276.

13. E. Witten and B. Zwiebach, IAS preprint IASSNS-HEP-91/100.

14. M. Li, UCSB preprint UCSBTH-91-47.

15. E. Witten, Phys. Rev. D44 (1991), 314. 